\title{
Complex Dynamics and Synchronization in a System of Magnetically Coupled Colpitts Oscillators
}

\author{
L. K. Kana, ${ }^{1,2}$ A. Fomethe, ${ }^{3}$ H. B. Fotsin, ${ }^{1}$ E. T. Wembe, ${ }^{2}$ and A. I. Moukengue ${ }^{2}$ \\ ${ }^{1}$ Laboratory of Electronics and Signal Processing, Department of Physics, Faculty of Science, \\ University of Dschang, P.O. Box 67, Dschang, Cameroon \\ ${ }^{2}$ Department of Physics, Faculty of Science, University of Douala, P.O. Box 24157, Douala, Cameroon \\ ${ }^{3}$ Laboratory of Mechanics and Modeling of Physical Systems, Faculty of Science, University of Dschang, \\ P.O. Box 67, Dschang, Cameroon \\ Correspondence should be addressed to L. K. Kana; kemgangkl@yahoo.com
}

Received 8 October 2016; Revised 13 February 2017; Accepted 23 February 2017; Published 10 April 2017

Academic Editor: Zhao Lu

Copyright (C) 2017 L. K. Kana et al. This is an open access article distributed under the Creative Commons Attribution License, which permits unrestricted use, distribution, and reproduction in any medium, provided the original work is properly cited.

\begin{abstract}
We propose the use of a simple, cheap, and easy technique for the study of dynamic and synchronization of the coupled systems: effects of the magnetic coupling on the dynamics and of synchronization of two Colpitts oscillators (wireless interaction). We derive a smooth mathematical model to describe the dynamic system. The stability of the equilibrium states is investigated. The coupled system exhibits spectral characteristics such as chaos and hyperchaos in some parameter ranges of the coupling. The numerical exploration of the dynamics system reveals various bifurcations scenarios including period-doubling and interior crisis transitions to chaos. Moreover, various interesting dynamical phenomena such as transient chaos, coexistence of solution, and multistability (hysteresis) are observed when the magnetic coupling factor varies. Theoretical reasons for such phenomena are provided and experimentally confirmed with practical measurements in a wireless transfer.
\end{abstract}

\section{Introduction}

In electronics and in nonlinear dynamics, oscillators involving inductors are always modeled without any coupling from external magnetic field. However, in certain experimental condition, non-physically coupled systems may interact.

In this paper, we study the effect of the magnetic coupling on the behavior of a Colpitts coupled oscillator. Coupled chaotic systems have become a topic of active research in many scientific areas such as chemistry, biology, optics, and communications. In particular, the synchronization of coupled chaotic systems has received a lot of attention since the early 1990s [1-3]. This has been motivated by potential application of chaotic signals in such areas as signal encryption and communications [4].

The classical Colpitts oscillator is widely used in electronic devices and communication system as a source of sinusoidal waveform with low harmonic content [5]. This oscillator has been extensively used in the investigation of various dynamical phenomena. With a special parameters settings this oscillator exhibits a rich dynamic behavior: period doubling and chaotic behavior [5-7]. As a chaotic generator it can be used to generate noise-like broadband signals. The oscillator is very flexible as the fundamental frequency can be tuned from several kilohertz to several gigahertz, that is, to the microwave range.

Hyperchaos is characterized as chaotic dynamics with more than one positive Lyapunov exponent (LE). Hyperchaos has attracted increasing attention from the scientific and engineering communities $[8,9]$. The interest devoted to hyperchaotic systems is motivated by their complex dynamics such as rich bifurcation, wider frequency bandwidths, and their potential application to information technologies, specifically to secure communication; hyperchaos has been firstly reported by Rössler [8], and the first circuit implementation of hyperchaos was realized by Matsumoto et al. [9]. Although the Colpitts oscillator is known to exhibit chaotic dynamics, it has also been shown to exhibit an hyperchaotic behavior when coupled in various configurations $[10,11]$. 


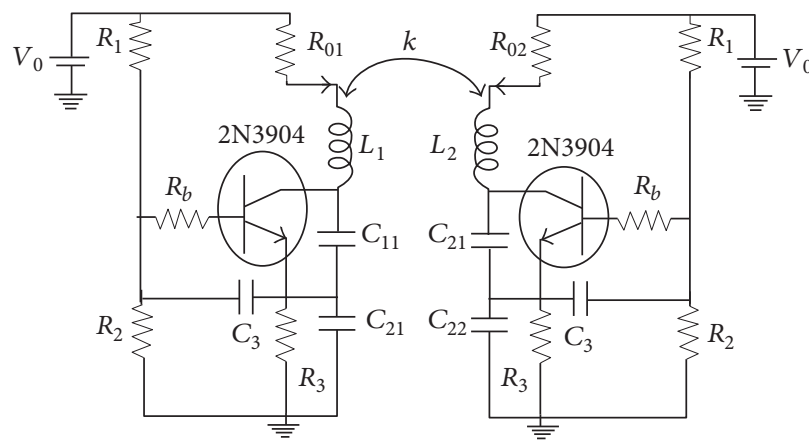

(a)

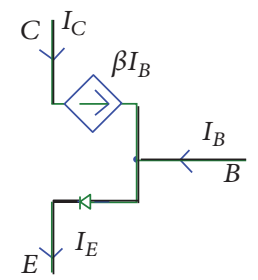

(b)

FIGURE 1: The electrical circuit of the system consisting of two mutually coupled Colpitts oscillators (a) and the circuit diagram of a bipolar junction transistor (BJT) (b). The circuit elements are $2 \mathrm{~N} 3904 ; V_{0}=10 \mathrm{~V} ; R_{0}=35 \Omega ; L_{j}=1.2 \mathrm{mH} ; C_{1 j}=C_{2 j}=470 \mathrm{nF} ; C_{3}=47 \mathrm{nF} ; R_{3}=$ $510 \Omega ; R_{b}=1 \Omega ;$ and $R_{1}=R_{2}=2 \mathrm{k} \Omega$.

In a complex system several attractors may coexist for a given set of system parameters. This coexistence is termed as multistability and has been found in almost all research areas of natural science, such as mechanics, electronics, biology, environmental science, and neuroscience [12,13]. Multistable systems are characterized by a high degree of complexity in dynamical behavior due to the interaction among the coexisting attractors [14].

Generally speaking, chaos synchronization can be referred to as behavior in which two or more coupled systems exhibit identical chaotic oscillations. For such dynamical systems, the loss of synchronization between the subsystems is strictly related to the Lyapunov exponents (LEs) of the global system. When the subsystems lose synchrony, a transition from chaotic to hyperchaotic behavior takes place; that is, at least two LEs become positive [11]. Several approaches to chaotic synchronization have been proposed in the literature, for example, adaptive control, active-backstepping design, active control, backstepping, sliding mode control, and self-adaptive backstepping [1-4]. In most of these methods, the controllers and the design approach are often very complex and may be difficult to achieve in practice.

In this paper, a new method is introduced. It has been experimentally and has been numerically characterized, leading to a simple, cheap, and easy technique for the study of dynamic and synchronization of Colpitts coupled oscillator. When compared with other circuit techniques applied to the chaotic oscillator [11], our approach requires a simple control circuitry (based on the magnetic coupling: wireless coupling). A previous work in this domain dealt with chaos control and synchronization of a system. It consisted of chaotic modified Van der Pol-Duffing oscillator, which is able to generate low frequency chaotic signal [15].

The idea underlying our approach is also applied to chaotic Colpitts oscillator. It demonstrates how it is possible to control and synchronize a system in a simple and effective way, with high frequency oscillations. This coupling gives a possibility of obtaining a rich and complex dynamic system. To the best of our knowledge, the magnetic coupling of two chaotic Colpitts oscillators has never been investigated.
Our aims in this work are the following: (a) extend the study of magnetic coupling to higher frequencies chaotic systems (they are more useful in practical secure communication systems), (b) investigate the conditions leading to practical synchronization of the coupled Colpitts circuits, and (c) present an electronic implementation of the magnetically coupled systems. The rest of the paper is organized as follows: In Section 2, the circuit of the magnetically coupled system is presented and an appropriate model is derived to describe the dynamical behavior of the global oscillator. Section 3 deals with the complex dynamical behavior of the proposed circuit. In Section 4, based on the Lyapunov theory factor, we demonstrate the effectiveness of the proposed controller. In Section 5, numerical and experimental simulation results are provided to illustrate the effectiveness and feasibility of the control and synchronization technique. Finally, conclusions are given in Section 6.

\section{Circuit Description of the Model and Differential Equations}

2.1. The Model. The Colpitts oscillator shown in Figure 1(a) contains a bipolar junction transistor 2N3904 (BJT), resistors, a feedback capacitor $C_{3}$, and a resonant circuit. The resonant circuit consists of an inductor and two capacitors $C_{1}$ and $C_{2}$. The model of the transistor is shown in Figure 1(b), with a nonlinear element (voltage-controlled) and a linear currentcontrolled current source. This oscillator has been used in electronic devices and communication systems, due to the fact that it can exhibit chaos [5].

2.2. State Equations. Let us consider the two magnetic core coils of Figure 1(a) and the magnetic coupling $k$ defined in [15]. The following set of differential equations are obtained by applying Kirchhoff's laws to the circuit shown in Figure 1(a):

$$
\begin{aligned}
c_{1 j} \frac{d V_{\mathrm{C} 1 j}}{d t} & =I_{L j}-\beta F\left(V_{\mathrm{C} 3 j}\right) \\
c_{2 j} \frac{d V_{\mathrm{C} 2 j}}{d t} & =I_{L j}+\frac{E_{\mathrm{th}}-V_{\mathrm{C} 3 j}}{R_{\mathrm{th}}}-V_{\mathrm{C} 2 j}\left(\frac{1}{R_{\mathrm{th}}}+\frac{1}{R_{b}}\right)
\end{aligned}
$$




$$
\begin{aligned}
c_{3 j} \frac{d V_{C 3 j}}{d t} & =\frac{E_{\mathrm{th}}-V_{C 3 j}-V_{C 2 j}}{R_{\mathrm{th}}}-F\left(V_{C 3 j}\right) \\
\frac{L_{j} d I_{L j}}{d t} & =V_{0}-R_{0} I_{L j}-V_{C 1 j}-V_{C 2 j}-M \frac{d i_{L_{3-j}}}{d t},
\end{aligned}
$$

where $F\left(V_{C 3}\right)=\left\{V_{C_{3 j}}-V_{\mathrm{BE}}\right.$, if $V_{C_{3 j}}>V_{\mathrm{BE}} ; 0$, if $V_{C_{3 j}} \leq$ $\left.V_{\mathrm{BE}}\right\}, R_{\mathrm{th}}=R_{1} R_{2} /\left(R_{1}+R_{2}\right), E_{\mathrm{th}}=\left(R_{2} /\left(R_{1}+R_{2}\right)\right) V_{0}$, and $\beta=150$ is the current gain (transistor gain) of the BJT. The relationship between the transistor gain and common base forward short-circuit gain of the transistor $\left(\alpha_{F}\right)$ is defined as $\beta=\alpha_{F} /\left(1-\alpha_{F}\right), j=1,2 ; M=k \sqrt{L_{j} \times L_{3-j}}$ is the mutual inductance $M$; if it is less than $L$, then the coupling magnetic $k=\left(1-(3 / 2)(2 x / l)^{2}\right)(l$ is the length of the coil and $x$ is the distance between the windings) [15] is also $-1 \leq k \leq 1$.

In our model (Figure 1), the values of electronic circuit components are $V_{0}=10 \mathrm{~V}, R_{0}=35 \Omega, L_{j}=1.2 \mathrm{mH}, C_{1 j}=$ $C_{2 j}=470 \mathrm{nF}, C_{3 j}=47 \mathrm{nF}, R_{3}=510 \Omega R_{b}=1 \Omega$, and $R_{1}=$ $R_{2}=2 \mathrm{k} \Omega$.

For the numerical investigation, an appropriate rescaling is introduced with the corresponding change of variables: $t=$ $\sqrt{L C_{1}} \tau ; x_{j}=V_{C_{1 j}} / V_{\mathrm{BE}}, y_{j}=V_{\mathrm{C}_{2 j}} / V_{\mathrm{BE}}, z_{j}=V_{\mathrm{C}_{3 j}} / V_{\mathrm{BE}}, w_{j}=$ $\left(I_{L} / V_{\mathrm{BE}}\right) \sqrt{L / C_{1 j}}$; one can rewrite (1) to obtain the following dimensionless set of differential equations:

$$
\begin{aligned}
& \dot{x}_{j}=w_{j}-a_{1} f\left(z_{j}\right) \\
& \dot{y}_{j}=w_{j}+\alpha_{0}\left(d-z_{j}\right)-\alpha_{1} y_{j} \\
& \dot{z}_{j}=\varepsilon \alpha_{0}\left(d-z_{j}-y_{j}\right)-\varepsilon a_{2} f\left(z_{j}\right) \\
& \dot{w}_{j}=e-b w_{j}-x_{j}-y_{j}-\frac{M}{L_{j}} \dot{w}_{3-j},
\end{aligned}
$$

where

$$
\begin{aligned}
f(z) & = \begin{cases}z_{j}-1 & \text { if } z_{j}>1 \\
0 & \text { if } z_{j} \leq 1\end{cases} \\
a_{2} & =\sqrt{\frac{L}{C_{1}}}=50.53 \\
a_{1} & =\beta a_{2}=7579.5,
\end{aligned}
$$

$$
\begin{aligned}
\alpha_{0} & =\frac{a_{2}}{R_{\mathrm{th}}}=0.05053 ; \\
\alpha_{1} & =\alpha_{0}+\frac{a_{2}}{R_{1}}=0.149 ; \\
d & =\frac{E_{\mathrm{th}}}{V_{\mathrm{BE}}}=7.14 ; \\
e & =\frac{V_{0}}{V_{\mathrm{BE}}}=14.28 ; \\
b & =\frac{R_{0}}{a_{2}}=0.69 \\
\varepsilon & =\frac{C_{1}}{C_{3}}=10 .
\end{aligned}
$$

2.3. Equilibrium Points and Their Stability. We consider the normalized system (2). The equilibrium points of this system can be found by solving the system of algebraic equations:

$$
\begin{array}{r}
w_{j}-a_{1}\left(z_{j}-1\right)=0 \\
w_{j}+\alpha_{0}\left(d-z_{j}\right)-\alpha_{1} y_{j}=0 \\
\varepsilon \alpha_{0}\left(d-z_{j}-y_{j}\right)-\varepsilon a_{2}\left(z_{j}-1\right)=0 \\
e-b w_{j}-x_{j}-y_{j}=0 .
\end{array}
$$

We obtain the equilibrium points $O\left(x_{0 j}, y_{0 j}, z_{0 j}, w_{0 j}\right)$, $(j=1,2)$ with

$$
\begin{aligned}
& x_{0 j}=\gamma_{0}\left(1-b a_{1}\right)+b a_{1}-d+e-\frac{a_{2}\left(1-\gamma_{0}\right)}{\alpha_{0}} \\
& y_{0 j}=d-\gamma_{0}+\frac{a_{2}-\gamma_{0} a_{2}}{\alpha_{0}} \\
& z_{0 j}=\gamma_{0} \\
& w_{0 j}=a_{1}\left(\gamma_{0}-1\right),
\end{aligned}
$$

where

$$
\gamma_{0}=1+\frac{\alpha_{0}(d-1)\left(\alpha_{1}-\alpha_{0}\right)}{\alpha_{0}\left(\alpha_{0}-a_{1}-\alpha_{1}\right)-\alpha_{1} a_{2}} .
$$

The equilibrium point $O$ does not depend on the control parameter $k$. By linearizing system (2) around this point, we obtain the following $8 \times 8$ Jacobian matrix:

$$
J=\left[\begin{array}{cccccccc}
0 & 0 & -a_{1} & 1 & 0 & 0 & 0 & 0 \\
0 & -\alpha_{1} & -\alpha_{0} & 1 & 0 & 0 & 0 & 0 \\
0 & -\varepsilon \alpha_{0} & -\varepsilon\left(\alpha_{0}+a_{2}\right) & 0 & 0 & 0 & 0 & 0 \\
-k_{0} & -k_{0} & 0 & -b k_{0} & -k k_{0} & -k k_{0} & 0 & -k k_{0} b \\
0 & 0 & 0 & 0 & 0 & 0 & -a_{1} & 1 \\
0 & 0 & 0 & 0 & 0 & -\alpha_{1} & -\alpha_{0} & 1 \\
0 & 0 & 0 & 0 & 0 & -\varepsilon \alpha_{0} & -\varepsilon\left(\alpha_{0}+a_{2}\right) & 0 \\
-k k_{0} & -k k_{0} & 0 & -k k_{0} b & -k_{0} & -k_{0} & 0 & -b k_{0}
\end{array}\right] .
$$




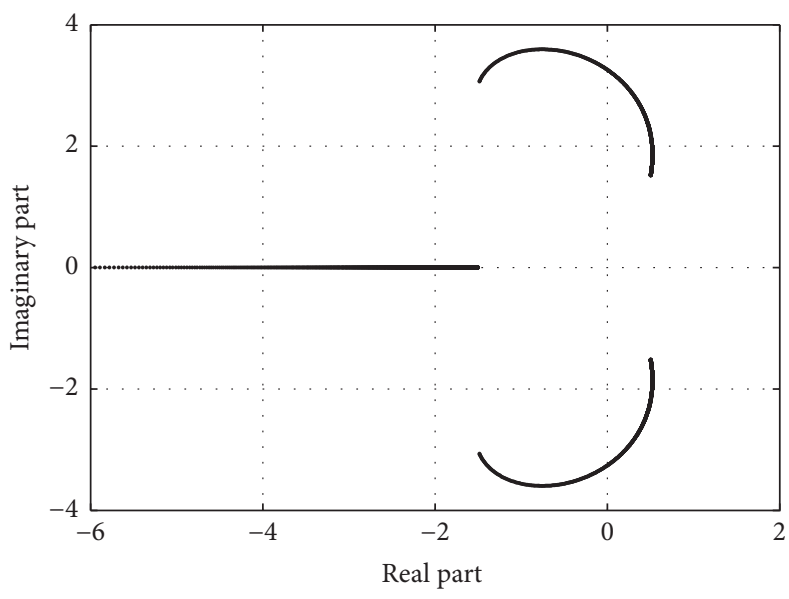

(a)

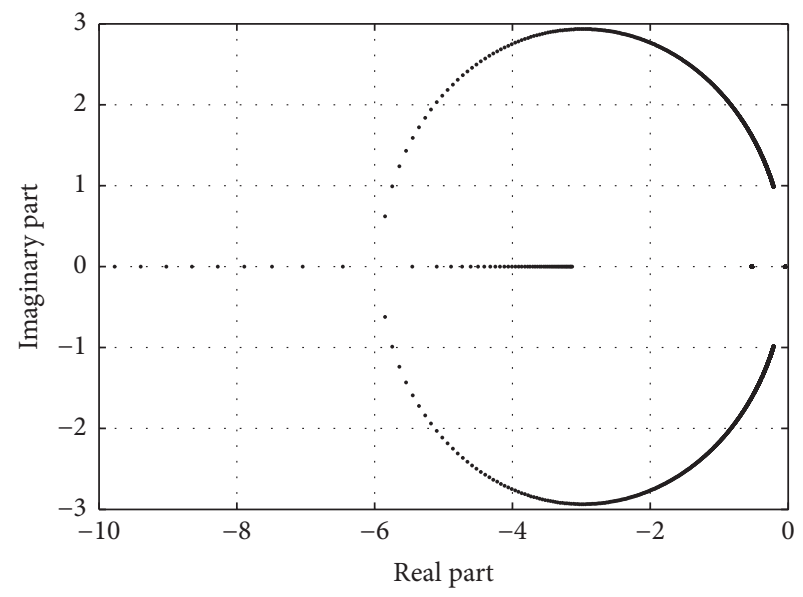

(b)

FIGURE 2: Representation of the eigenvalues, solution of the characteristic equation (11), in the complex plane $(\operatorname{Re}(\lambda), \operatorname{Im}(\lambda))$ when $f(z)=0$ (a) and when $f(z)=z-1$ (b).

The stability of the equilibrium point $O$ can be analyzed by solving the characteristic equation $\operatorname{det}\left(M_{J}-\lambda I_{d}\right)=0$, where $I_{d}$ is the $8 \times 8$ identity matrix. Then, the eigenvalues are obtained by solving the following characteristic equation:

$$
\begin{aligned}
\lambda^{8}+ & A_{7} \lambda^{7}+A_{6} \lambda^{6}+A_{5} \lambda^{5}+A_{4} \lambda^{4}+A_{3} \lambda^{3}+A_{2} \lambda^{2} \\
+ & A_{1} \lambda+A_{0}=0
\end{aligned}
$$

with

$$
\begin{aligned}
A_{7} & =2\left(b+\delta_{1}\right) \\
A_{6} & =\left[4+2 \delta_{0}+\delta_{1}\left(4 b+\delta_{1}\right)\right]+b^{2} k \\
A_{5} & =2 \delta_{1}\left(b \delta_{1}+3\right)+2 \delta_{0}\left(2 b+\delta_{1}\right)+2 q+k_{0} b[4 \\
& \left.+2 b \delta_{1}+\delta_{0}\right] \\
A_{4} & =2 q+2 a_{1} \varepsilon \alpha_{0}+\delta_{0}\left(6+4 b \delta_{1}+\delta_{0}\right)+2 \delta_{1}\left(\delta_{1}+q\right) \\
& +k_{0}\left[4+2 b q+b \delta_{1}\left(6+b \delta_{1}\right)+b \delta_{0}\left(2 b+\delta_{1}\right)\right] \\
A_{3} & =2 \delta_{0}\left(2 \delta_{1}+q+b \delta_{0}+\delta_{1} a_{1} \varepsilon \alpha_{0}\right)+k_{0}\left[2 q\left(2+b \delta_{1}\right)\right. \\
& \left.+b\left(2+a_{1} \varepsilon \alpha_{0}+4 \delta_{0}+2 \delta_{1}^{2}\right)+\delta_{1} b^{2}\left(1+\delta_{0}\right)+4 \delta_{1}\right] \\
A_{2} & =2 \delta_{0}\left(\delta_{0}+a_{1} \varepsilon \alpha_{0}\right)+k_{0}\left[\left(\delta_{1}+q+b \delta_{1}\right)^{2}\right. \\
& \left.+2\left(\delta_{0}+a_{1} \varepsilon \alpha_{0}\right)\left(2+b \delta_{1}\right)\right] \\
A_{1} & =k_{0}\left(\delta_{0}+a_{1} \varepsilon \alpha_{0}\right)\left(\delta_{1}+q+2 b \delta_{0}\right) \\
A_{0} & =k_{0}\left(\delta_{0}+a_{1} \varepsilon \alpha_{0}\right)^{2},
\end{aligned}
$$

where

$$
\begin{aligned}
& k_{0}=\frac{1}{\left(1-k^{2}\right)}, \\
& \delta_{0}=\alpha_{1} \varepsilon a_{2}+\left(\alpha_{1}-\alpha_{0}\right) \varepsilon \alpha_{0}, \\
& \delta_{1}=\alpha_{1} \varepsilon a_{2}+\left(\alpha_{1}+a_{1}-\alpha_{0}\right) \varepsilon \alpha_{0}, \\
& \delta_{3}=\alpha_{1}+\varepsilon\left(\alpha_{0}+a_{2}\right) .
\end{aligned}
$$

Using the Routh-Hurwitz criteria on system (8), it appears that its trivial fixed point is stable if the following condition is satisfied:

$$
\begin{aligned}
& \frac{\eta_{0}\left(\left(C_{2} / C_{1}\right) \eta_{1}-\eta_{0}\right)-\eta_{1}\left(A_{0} C_{1}-\left(C_{3} / C_{1}\right) \eta_{0}\right)}{C_{1} \eta_{0}-C_{2} \eta_{1}}-A_{0} \\
& \quad>0,
\end{aligned}
$$

where

$$
\begin{aligned}
& C_{1}=A_{3} A_{7}-A_{4} A_{7}^{2}-A_{5}^{2}+A_{6} A_{7} A_{5}, \\
& C_{2}=A_{1} A_{7}-A_{2} A_{7}^{2}-A_{3} A_{5}+A_{6} A_{7} A_{3}, \\
& C_{3}=-A_{0} A_{7}^{2}-A_{5} A_{1}+A_{6} A_{7} A_{1}, \\
& \eta_{0}=\left(A_{5}-A_{6} A_{7}\right) C_{3}-\left(A_{1}-A_{7} A_{2}\right) C_{1}, \\
& \eta_{1}=\left(A_{5}-A_{6} A_{7}\right) C_{2}-\left(A_{3}-A_{4} A_{7}\right) C_{1} .
\end{aligned}
$$

(i) If $z_{j}>1, f(z) \neq 0\left(a_{1}=0\right.$ and $\left.a_{2}=0\right)$, condition (11) can be solved choosing $k$ as the control parameter and keeping the other parameters constant; for $0<k<1$ this condition is not satisfied and the equilibria point $O$ is unstable saddle focus. Figure 2(a) illustrated such assertion, where we plot in the complex plane 
$(\operatorname{Re}(\lambda) ; \operatorname{Im}(\lambda))$ the imaginary part of eigenvalues as a function of their real part. We observe that, for $0<$ $k<1$, there exists the real part of the corresponding eigenvalues which is positive.

(ii) If $z_{j} \leq 1, a_{1}=a_{2}=0$, condition (11) is satiated for all values for $k$. In Figure 2, we show the eigenvalues in the complex plane $(\operatorname{Re}(\lambda) ; \operatorname{Im}(\lambda))$. Equation (8) is solved using the Newton-Raphson algorithm for the range of parameter $0<k<1$ : the real parts of the eigenvalues $(\lambda)$ are all negative; then the equilibrium point is a stable spiral point.

Let us consider the coefficient $k$ as the control parameter, since it can vary through experiment with the distance between the magnetic core coils. Applying the condition for the occurrence of the Hopf bifurcation: in (8) there exists a pair of purely imaginary complex eigenvalues and $(d \lambda / d k)_{k=k c} \neq 0$, where $k_{c}$ is called the critical value for the onset of the bifurcation. In the case of $f(z) \neq 0$, the critical values of the parameter control are obtained by solving the equation:

$$
\left(17 \eta_{1}^{2}-12 \eta_{3}\right)^{3}+(2 \eta)=0
$$

with

$$
\begin{aligned}
& \eta_{1}=A_{4}-\frac{3}{8} A_{6}^{2}, \\
& \eta_{2}=\frac{A_{6}}{2}\left(A_{4}-\frac{A_{6}^{2}}{4}\right)-A_{2}, \\
& \eta_{3}=\frac{A_{6}^{2}}{16}\left(A_{4}-\frac{A_{6}^{2}}{16}\right)+A_{6}\left(1-\frac{A_{2}}{4}\right) .
\end{aligned}
$$

The critical value can be obtained by making recourse to numerical methods. We have one critical value $k_{c}=$ 0.8056001; the corresponding eigenvalues are $\lambda_{1}=-3.6983$; $\lambda_{2}=3.2771 i ; \lambda_{3}=-3.2771 i ; \lambda_{4}=0.51211+1.577 i$; $\lambda_{5}=0.51211-1.577 i ; \lambda_{6}=-1.5553 ; \lambda_{7}=-505.81$; and $\lambda_{8}=-505.81$.

\section{Dynamical Behavior of the Mutually Coupled Colpitts Systems}

3.1. Bifurcation Analysis. As we mentioned above, one of the main advantages of this circuit for experimental applications is the richness of its dynamics. Here, the types of behaviors are identified using two indicators. The first indicator is the bifurcation diagram, the second being the graph of Lyapunov exponent's spectra [7]. In order to define different types of behaviors in our model, system (4) is solved numerically through the fourth-order Runge-Kutta integration algorithm. For each set of parameters used in this work, the time step is always $\Delta t=0.005$ and the simulations are done with variables and constant parameters in extended mode [16, 17]. For each case, system (2) is integrated for a sufficiently long time and the transient is discarded with the time step being always $\Delta t=0.005$. According to [18], the dynamics of the nonlinear system (2) can be classified in terms of the Lyapunov exponents $\lambda_{i}(i=1,2,3,4,5,6,7,8)$ as follows:

(1) For an equilibrium point, $\lambda_{i}<0$.

(2) For a limit cycle (periodic orbits), $\lambda_{1}=0$, $\lambda_{2,3,4,5,6,7,8}<0$.

(3) For 2-torus (quasiperiodic orbits), $\lambda_{1,2}=0$, $\lambda_{3,4,5,6,7,8}<0$.

(4) For 3-torus, $\lambda_{1,2,3}=0, \lambda_{4,5,6,7,8}<0$.

(5) For chaotic orbits, $\lambda_{1}>0, \lambda_{2}=0$, and $\lambda_{3,4,5,6,7,8}<0$.

(6) For hyperchaotic orbits, $\lambda_{1,2}>0, \lambda_{3}=0$, and $\lambda_{4,5,6,7,8}<0$.

The evolution process of the system is analyzed precisely by the means of the Lyapunov exponents spectrum, bifurcation diagrams, and phase portraits when varying the parameter $k$ in tiny step $0<k<0.9$; the other parameters are kept constant (the values defined in Section 2). The graph of the Lyapunov exponent spectrum and its corresponding bifurcation diagrams are depicted, in Figure 4. The bifurcation diagram is obtained by plotting local maxima of the states $x ; y ; z$; and $w$. It can be seen that the bifurcation diagram coincides well with the spectrum of the Lyapunov exponents. In Figures 3 and 4, the following scenarios emerge when monitoring the control parameter $k$ : hyperchaos $\rightarrow$ quasiperiodic $\rightarrow$ hyperchaos $\rightarrow$ chaos. Figure 5 shows some numerical phase portraits, obtained by Matlab simulation (1) and PSpice (2) analysis, respectively, when $0.6<k<$ 1. Figure $5(\mathrm{a})$ shows a quasiperiodic attractor $(k=0.67)$. Figures 5(b) and 5(c) show, respectively, hyperchaos and chaos $(k=0.8$ and $k=0.9)$. Practically, the distance between two magnetically coupled Colpitts systems or the position or type of magnetic coupling core could have drastic effects on the oscillators dynamics, even if the number of oscillators is increased. This is an interesting behavior in the sense of control of the collective dynamics of magnetically coupled nonlinear oscillators [15].

3.2. Multistability or Coexistence of Attractors. Generally, multistable systems are characterized by a high degree of complexity in dynamical behavior due to the interaction among the coexisting attractors. First of all, the dynamics of a multistable system are extremely sensitive to initial conditions. Due to the coexistence of different attractors and complex fractal basin boundary structures very small perturbations of the initial state may influence the final attractor. Second, the qualitative behavior of the system often changes under the variation of the parameters system. Similarly, attractors exist only in small intervals of the parameters system. A slight change in a control parameter may cause a rapid change in the number and type of coexisting attractors. Third, multistable systems are extremely sensitive to noise. Noise may cause a popping process between various attractors. As an example of the system which possesses multistability, we consider the system described by (2) and consider $k$ as a control parameter. In Figure 6 we present the bifurcation diagrams of system (2), with the values of 


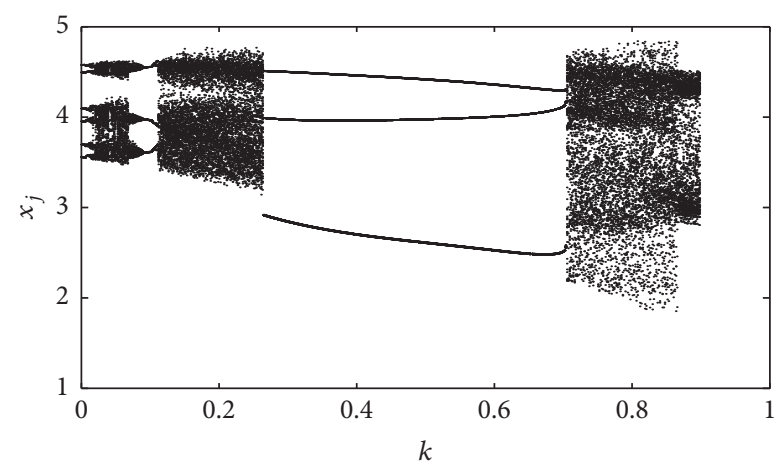

(a)

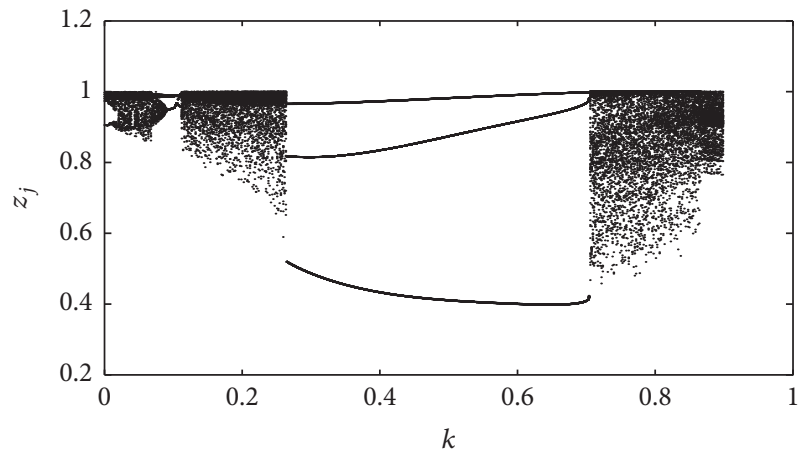

(c)

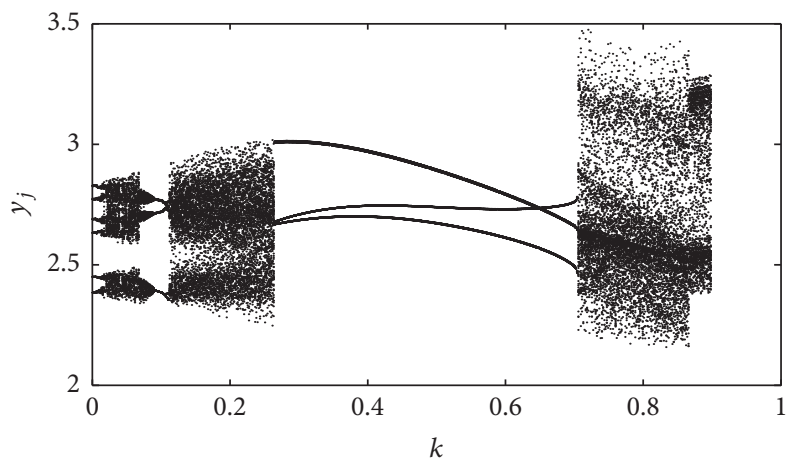

(b)

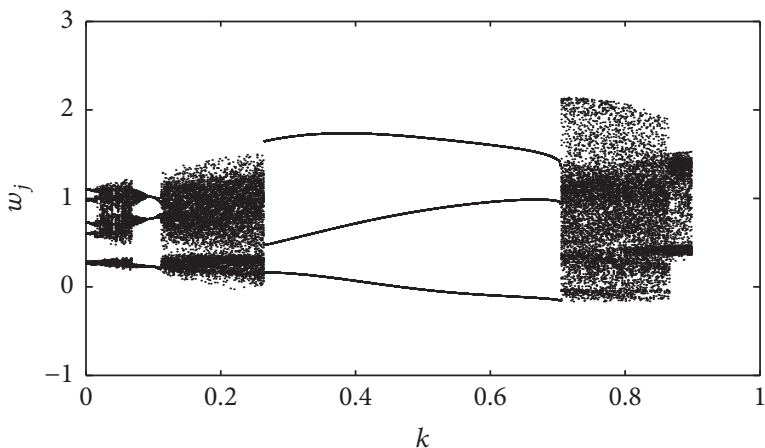

(d)

Figure 3: Bifurcation diagram of magnetically coupled Colpitts systems (2) showing the coordinates $x_{j}, y_{j}, z_{j}$, and $w_{j}(j=1,2)$. In terms of the control parameter $k$, the other system parameters are $a_{2}=50.53, a_{1}=150 a_{2}, \alpha_{0}=0.05053, \alpha_{1}=0.15, \varepsilon=10, d=3.57, e=7.4$, and $b=0.69$.
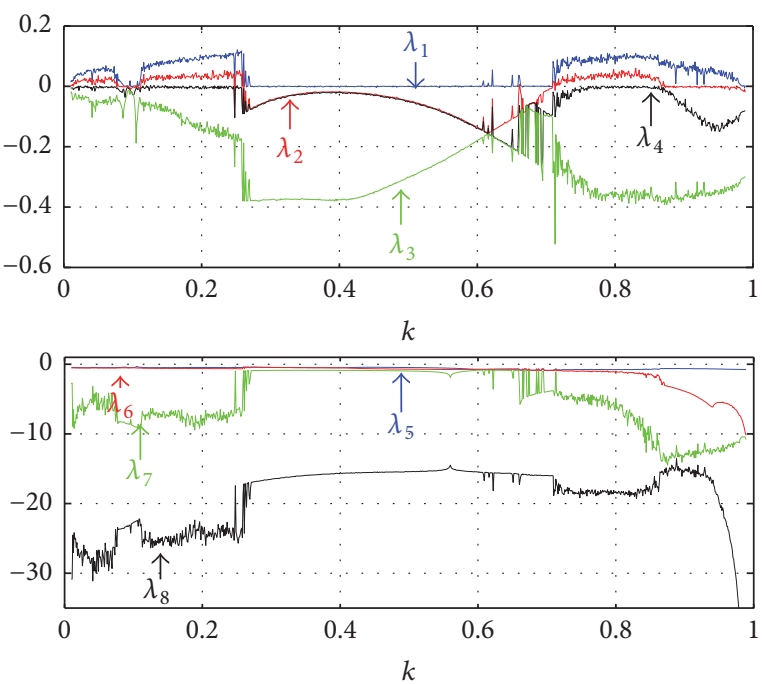

FIGURE 4: Lyapunov exponents spectrum of magnetically coupled Colpitts systems (2) versus the control parameter $k$. The rest of the parameters are those of Figure 3.

magnetic coupling $k$ on the horizontal axes. In the blue region of Figure 6 the values of $x_{j}$ have been calculated for the increasing values of $k$ while in the red region for the decreasing values of $k$, period-3 and chaos coexist for $0.08<$ $k<0.12$; chaos and period -5 coexist for $0.234<k<0.264$; period-3 and chaos coexist for $0.264<k<0.46,0.48<k<$ 0.54 , and $0.545<k<0.7$; period-3 (increases) and period-5 coexist for $0.46<k<0.48$; and period- 3 and period- 6 coexist for $0.54<k<0.545$.

For instance, In Figure 7 we present the coexistence of two different attractors, which can be obtained under the initial conditions $x_{1}=0.004, y_{1}=0.004, z_{1}=-0.09, w=$ $0.0032 ; x 2= \pm 0.004 ; y=0.004, z_{2}=0.09 ; w_{2}=0.0032$.

3.3. Complex Transient Chaos and Hyperchaos. The appearance of chaos and hyperchaos on finite time scales is known as transient chaos and transient hyperchaos. In our system, the appearance of periodic or chaos or hyperchaos motion strongly depends not only on the circuit parameters but also on the initial conditions. When the magnetic coupling of the stimulus is selected as $k=0.1$, a phenomenon of transient chaos is generated in the periodically coupled Colpitts oscillator (the trajectories of the system have a transition from transient chaotic to steady periodic behaviors with time evolutions). Figure 8(b) displays the time-domain wave form of variable $x_{j}$, where chaotic attractor is located in the time interval $[0,0.935]$ and then a limit cycle with period-3 forms after $t>935$; Figure 8(b) depicts two phase portraits of chaos and period-3 attractors in different phase planes.

The transient hyperchaos phenomenon exists when $k=$ 0.6: Figure 8 shows the time-domain waveforms and the 
(1)

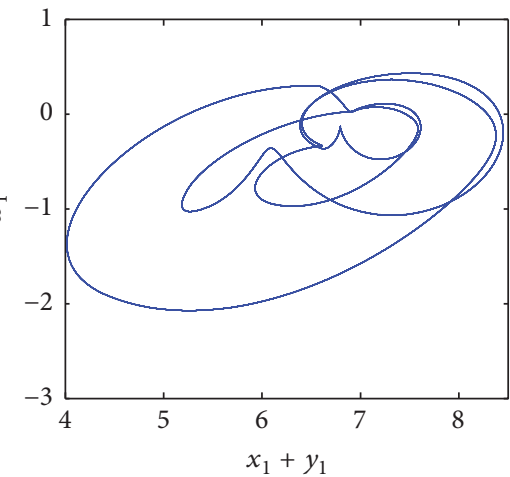

(2)

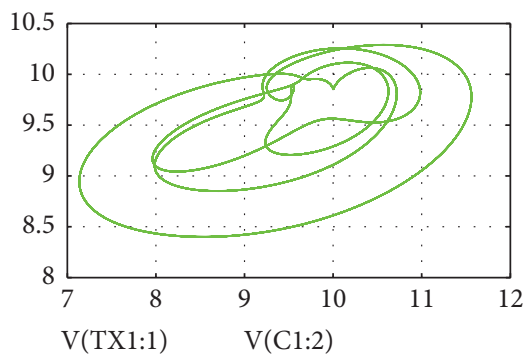

(a)
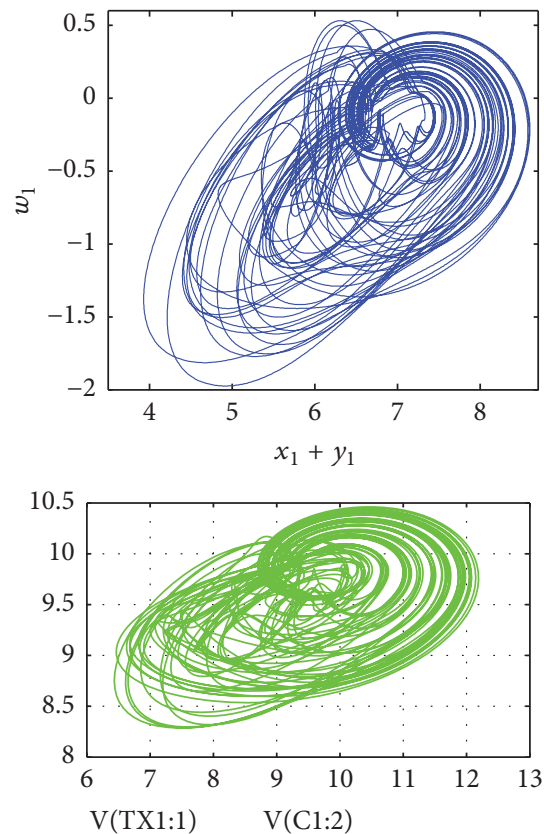

(b)
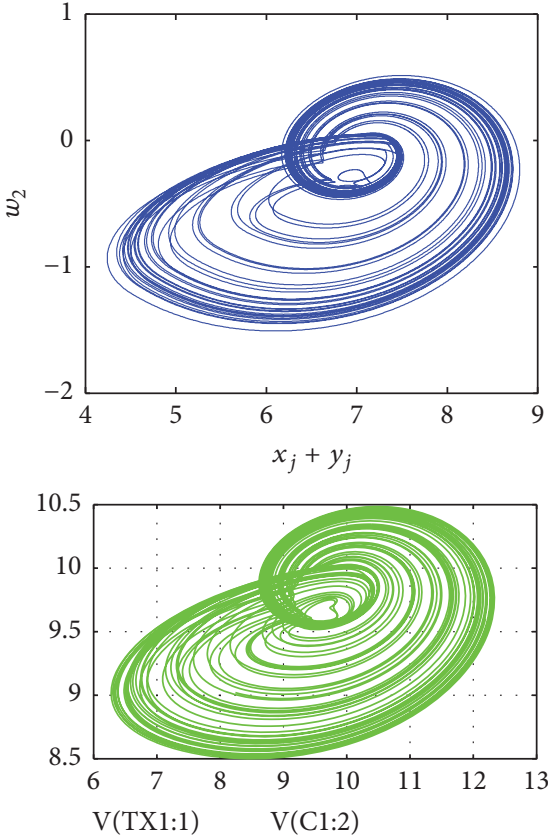

(c)

FIGURE 5: Qualitative comparison of numerical phase forms (1) and experimental (PSpice) phase portraits (2) of the oscillator, obtained for same specific values of the control parameter $k$ : (a) a quasiperiodic attractor for $k=0.67$; hyperchaotic attractor for $k=0.8$; and chaotic attractor for $k=0.9$.

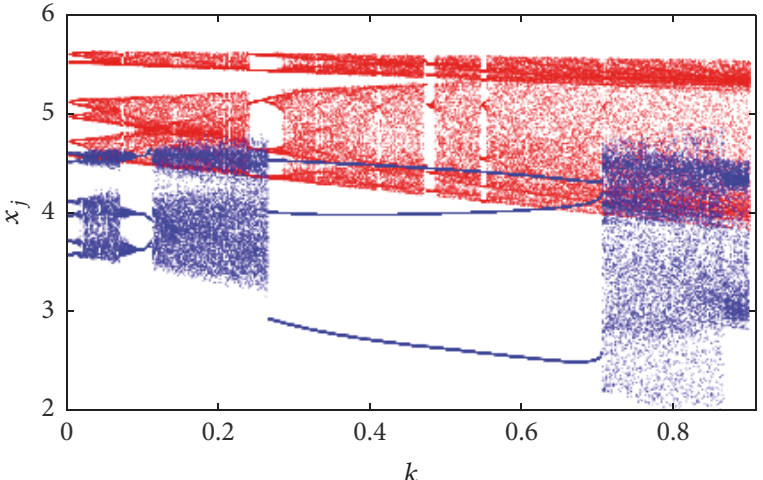

FIGURE 6: Bifurcation diagrams for (blue) increasing and (red) decreasing of magnetic coupling.

phase portrait of the hyperchaotic attractor combined with period-3, where hyperchaotic attractor is located in the time interval $[0 ; 585]$ and then a limit cycle with period-3 forms after $t>585$. Figure 9(a) displays the time-domain wave form of variable $x_{j}$, where hyperchaotic attractor is located in the time interval $[0,550]$ (Figure 9(b)), and then a limit cycle with period-3 forms after $t>550$ (Figure 9(c)).

\section{Synchronization of Two Modified Colpitts Oscillators}

4.1. Case of Two Identical Oscillators. Although the coupling scheme implemented here is bidirectional, we need to define a master (drive) and a slave (response) system. We consider here that the drive system is given by the following set of coupled differential equations:

$$
\begin{aligned}
\dot{x}_{1} & =w_{1}-a_{1} f\left(z_{1}\right), \\
\dot{y}_{1} & =w_{1}+\alpha_{0}\left(d-z_{1}\right)-\alpha_{1} y_{1}, \\
\dot{z}_{1} & =\varepsilon \alpha_{0}\left(d-z_{1}-y_{1}\right)-\varepsilon a_{2} f\left(z_{1}\right), \\
\dot{w}_{1} & =e-b w_{1}-x_{1}-y_{1}+k \dot{w}_{2} .
\end{aligned}
$$

According to our aim, we define the controlled response system as the following set of differential equations:

$$
\begin{aligned}
\dot{x}_{2} & =w_{2}-a_{1} f\left(z_{2}\right), \\
\dot{y}_{2} & =w_{2}+\alpha_{0}\left(d-z_{2}\right)-\alpha_{1} y_{2}, \\
\dot{z}_{2} & =\varepsilon \alpha_{0}\left(d-z_{2}-y_{2}\right)-\varepsilon a_{2} f\left(z_{2}\right), \\
\dot{w}_{2} & =e-b w_{2}-x_{2}-y_{2}+k \dot{w}_{1},
\end{aligned}
$$

where $k$ is the control parameter. We define the error system as the difference between the signals from the master and the slave system as $e_{x}=x_{1}-x_{2}, e_{y}=y_{1}-y_{2}, e_{z}=z_{1}-z_{2}$, and $e_{w}=w_{1}-w_{2}$. By considering the time derivatives of the error signals together with (15) and (16), we obtain the error dynamics system:

$$
\begin{aligned}
\dot{e}_{x} & =e_{w}-a_{1} e_{z}, \\
\dot{e}_{y} & =e_{w}-\alpha_{0} e_{z}+\alpha_{1} e_{y}, \\
\dot{e}_{z} & =-e_{z}\left(\alpha_{0}+a_{2}\right) \varepsilon-\varepsilon \alpha_{0} e_{y}, \\
\gamma \dot{e}_{w} & =-b e_{w}-e_{x}-e_{y},
\end{aligned}
$$

where $\gamma=1-k$. 


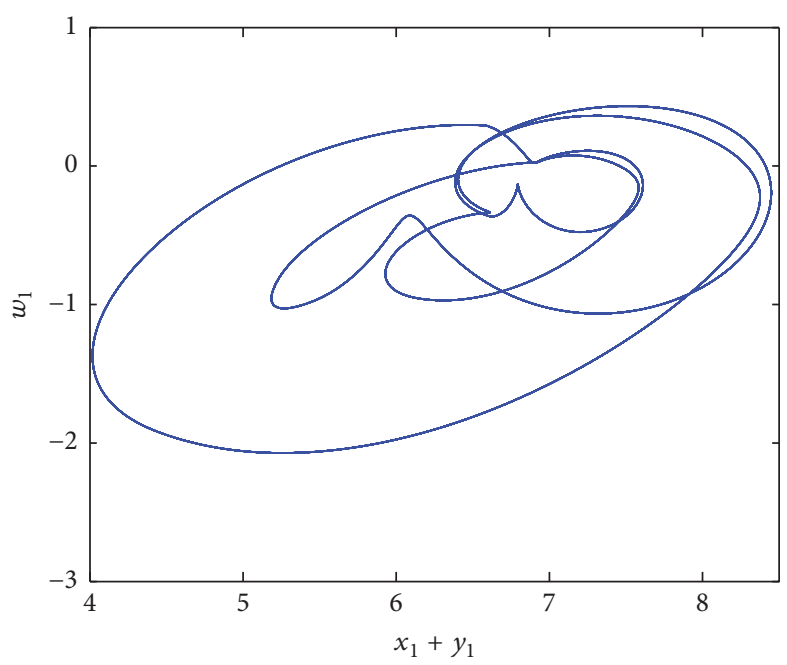

(a)

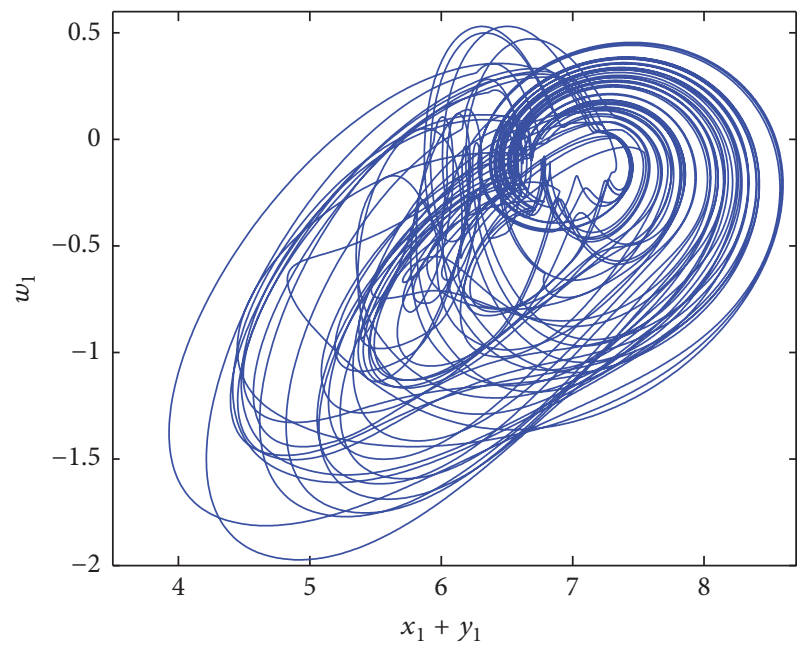

(b)

FIGURE 7: Coexistence of two different attractors, 3-period and hyperchaos, for $k=0.75$ and initial conditions $\left(x_{01}, y_{01}, z_{01}, w_{01}, x_{02}, y_{02}, z_{02}\right.$, $\left.w_{02}\right)=(0,0,0,0.2,0,0,0, \pm 0.35)$.

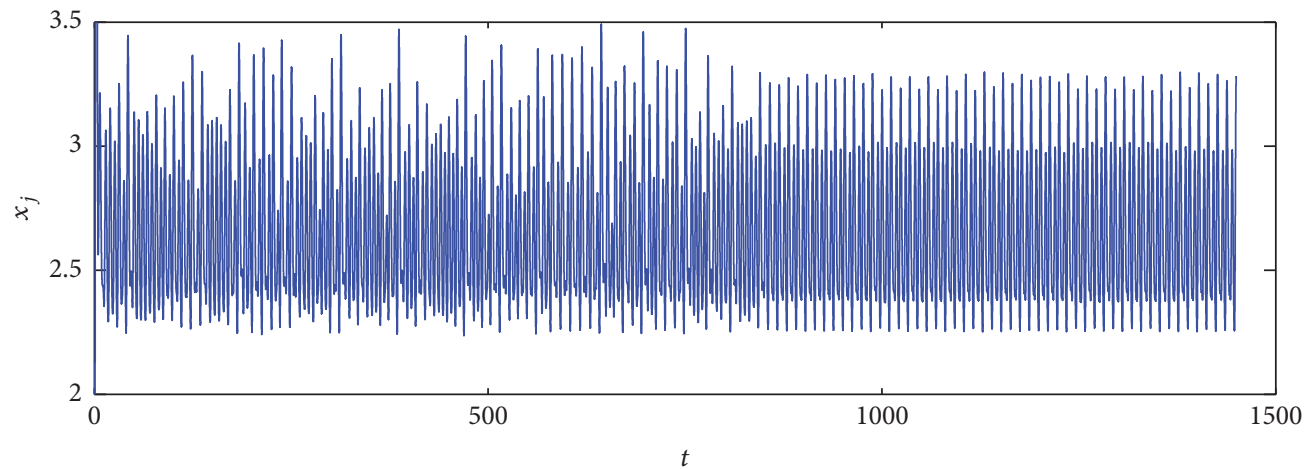

(a)

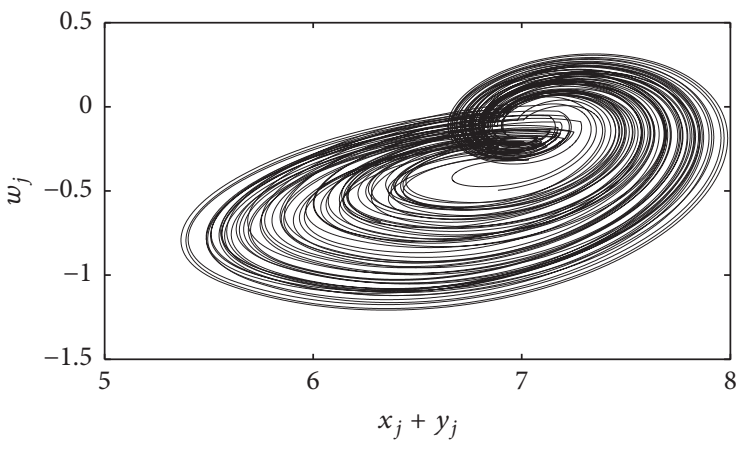

(b)

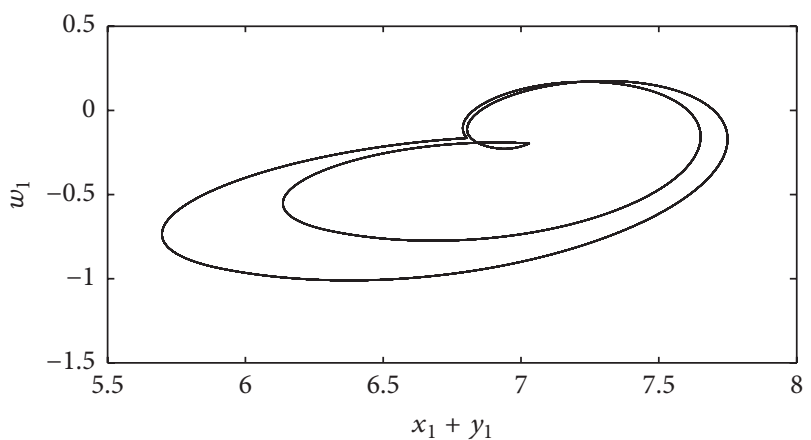

(c)

FIGURE 8: Transition from chaotic to period doubling: time-domain waveform of variable $x_{i}$ (a), the phase portrait in the time interval $t<825$ (b), and the phase portrait in the time interval $t>825$ (c) for initial conditions $x_{01}=0.004 ; y_{01}=0.004 ; z_{01}=-0.09 ; w_{01}=0.0032 ; x_{02}=$ $-0.034 ; y_{02}=0.008 ; z_{02}=0.009 ; w_{02}=0.1032$ when $k=0.1$.

It is clear that the synchronization problem is replaced by the equivalent problem of stabilizing (17) using a suitable choice of the control law $k$.

Proposition 1. The synchronization problem for the magnetically coupled Colpitts system is to achieve the asymptote of the zero solution of the error system (17) in the sense that $\left\|e_{i}(t)\right\| \rightarrow$ 0 , as $\rightarrow \infty$ where $(i=x ; y ; z ; w)$.

Proof. Consider a Lyapunov function candidate in the following form:

$$
V=\frac{1}{2}\left[\left(\lambda_{1} e_{x}+\lambda_{4} e_{w}\right)^{2}+e_{y}^{2}+\lambda_{3}^{2} e_{z}^{2}\right] .
$$




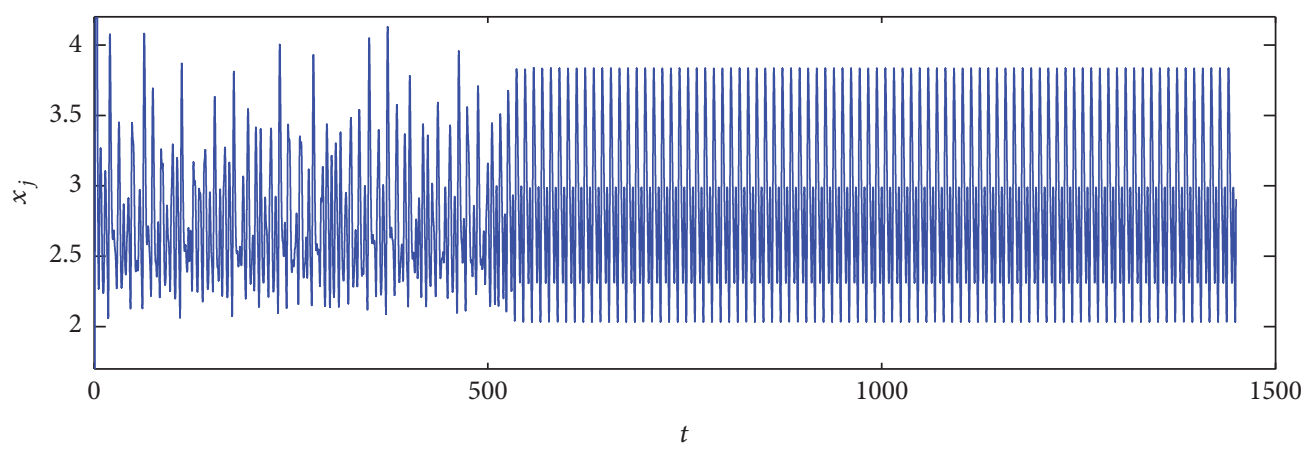

(a)

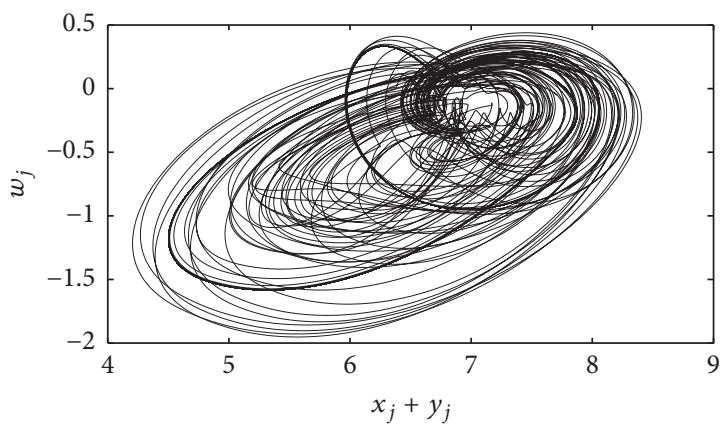

(b)

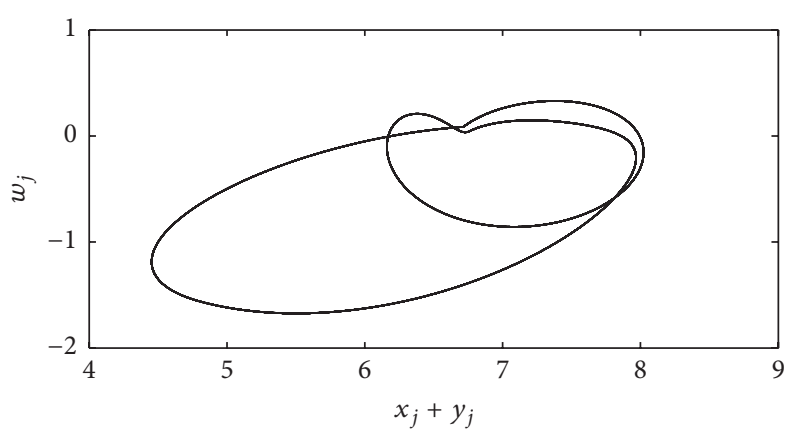

(c)

FIGURE 9: Transition from hyperchaos to period doubling: time-domain waveform of variable $x_{i}(\mathrm{a})$, the phase portrait in the time interval $t<500$ (b), and the phase portrait in the time interval $t>550$ (c) for initial conditions $x_{01}=0.004 ; y_{01}=0.004 ; z_{01}=-0.09 ; w_{01}=0.0032$; $x_{02}=-0.034 ; y_{02}=0.008 ; z_{02}=0.009 ; w_{02}=0.1032$ when $k=0.7$.

If the following condition holds

$$
16 \alpha_{1} b_{0}^{2} \alpha_{0} a_{1}=\left(\alpha_{0}+a_{2}\right)\left(4 c_{0} \alpha_{1} a_{1}-\alpha_{0}\right) c_{1}
$$

with $c_{1}=c_{0}+4$, the time derivative of function (18) with respect to the system of errors equations (17) satisfies

$$
\dot{V}=-\left(\lambda_{1} e_{x}+e_{y}+\lambda_{3} e_{z}+\lambda_{4} e_{w}\right)^{2}
$$

with $\lambda_{1}^{2}=\left(2 \alpha_{1} b-1\right)+\sqrt{\left(2 \alpha_{1} b-1\right)^{2}+48 \alpha_{1}^{2}} ; \lambda_{3}^{2}=$ $\lambda_{1}^{2} a_{1}^{2} / 16 \alpha_{1} \varepsilon\left(\alpha_{0}+a_{2}\right)$; and $\lambda_{4}^{2}=16 \alpha_{1}^{2} / \lambda_{1}^{2}$.

Since $V$ is positive definite function and $\dot{V}$ is a negative function, it follows that the origin equilibrium point of system (17) is asymptotically stable. Therefore, from (20) we can easily show that $e_{i}^{2}(t)(i=x ; y ; z ; w)$ is integrable with respect to time, for any initial condition $e_{i}(t) \rightarrow 0$ as $t \rightarrow \infty(i=$ $x ; y ; z ; w)$. This result concludes the proof.

4.2. Case of Nonidentical Oscillator (Mismatched Oscillators). In this section, we consider two oscillators $\mathrm{O} 1$ and $\mathrm{O} 2$ with the different magnetic core coils, coupled via magnetic coupling $k$ (Figure 1). In this case the overall system is given by

$$
\begin{aligned}
& \dot{x}_{1}=w_{1}-a_{1} f\left(z_{1}\right), \\
& \dot{y}_{1}=w_{1}+\alpha_{0}\left(d-z_{1}\right)-\alpha_{1} y_{1},
\end{aligned}
$$

$$
\begin{aligned}
& \dot{z}_{1}=\varepsilon \alpha_{0}\left(d-z_{1}-y_{1}\right)-\varepsilon a_{2} f\left(z_{1}\right), \\
& \dot{w}_{1}=e-b w_{1}-x_{1}-y_{1}-k \sqrt{\frac{L_{1}}{L_{2}}}\left(e-b w_{2}-x_{2}-y_{2}\right), \\
& \dot{x}_{2}=w_{2}-a_{1} f\left(z_{2}\right), \\
& \dot{y}_{2}=w_{2}+\alpha_{0}\left(d-z_{2}\right)-\alpha_{1} y_{2}, \\
& \dot{z}_{2}=\varepsilon \alpha_{0}\left(d-z_{2}-y_{2}\right)-\varepsilon a_{2} f\left(z_{2}\right), \\
& \dot{w}_{2}=e-b w_{2}-x_{2}-y_{2}-k \sqrt{\frac{L_{1}}{L_{2}}}\left(e-b w_{1}-x_{1}-y_{1}\right) .
\end{aligned}
$$

A three-parameter diagram showing regions of synchronization (blue) in the $\left(L_{2} ; L_{1} ; k\right)$ space computed, with the other parameter values stated in Section 2, is presented in Figure 10.

\section{Numerical and Experimental Simulations of the Controlled Synchronization}

5.1. Numerical Study. In this section, we present numerical simulation results to verify the effectiveness of the controller. In all cases, we select the parameters $a_{2}=50.53, a_{1}=150 a_{2}$, 


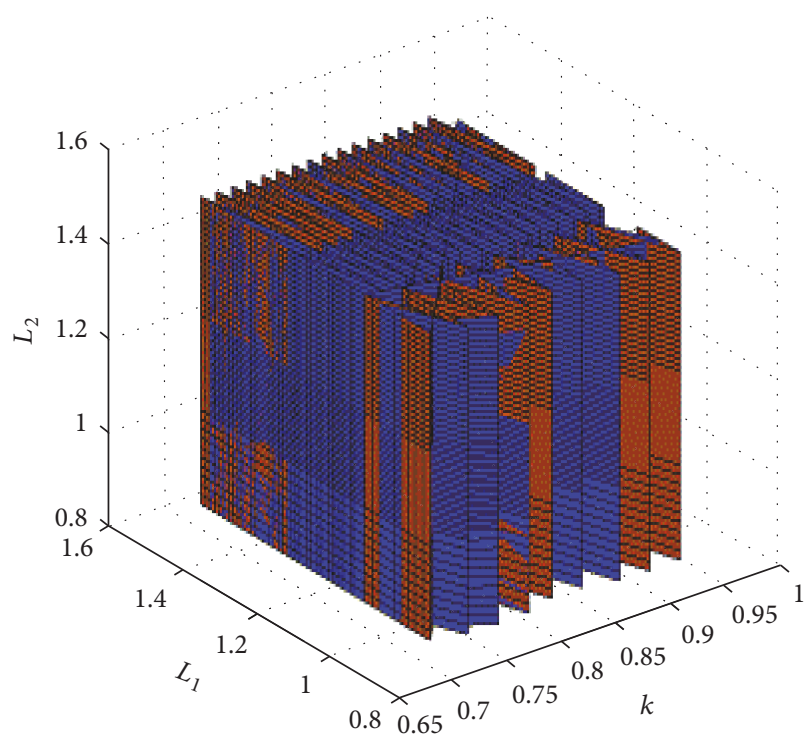

FIGURE 10: Three parameters of system $\left(L_{2} ; L_{1} ; k\right)$, showing regions of synchronization (blue) and unsynchronization (brown) with the initial conditions $x_{01}=0.004 ; y_{01}=0.004 ; z_{01}=-0.09 ; w_{01}=0.0032 ; x_{02}=-0.004 ; y_{02}=0.004 ; z_{02}=0.09 ; w_{02}=0.0032$.

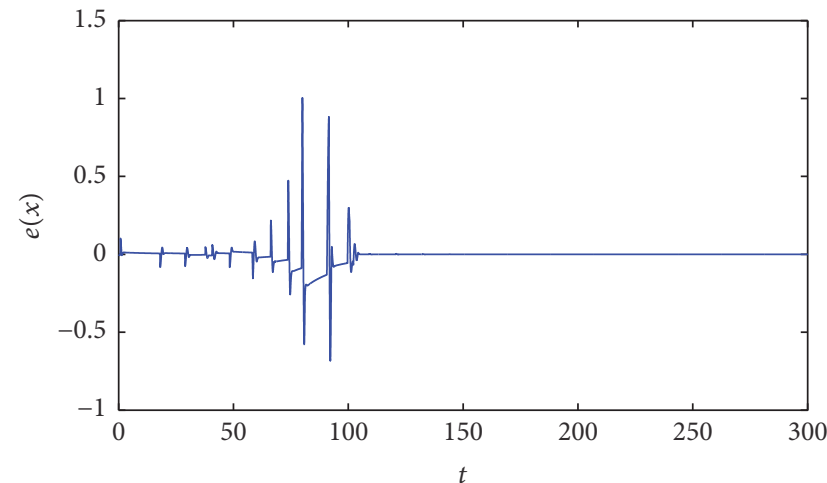

(a)

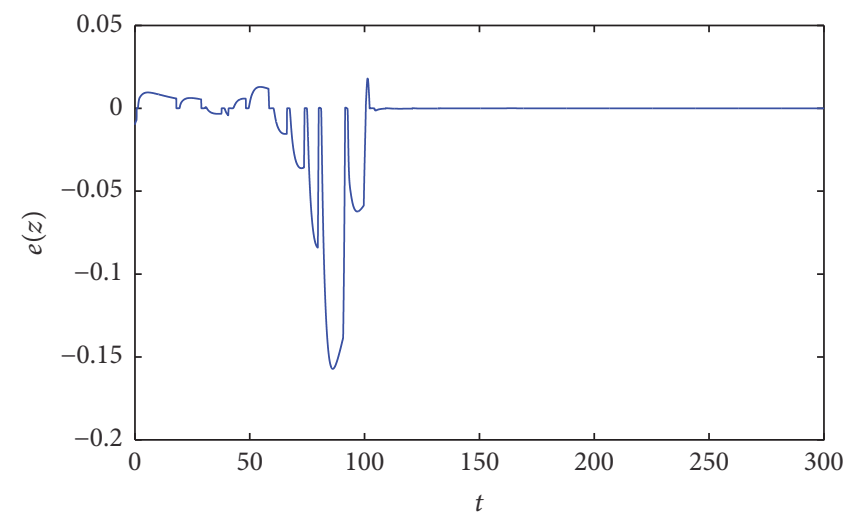

(c)

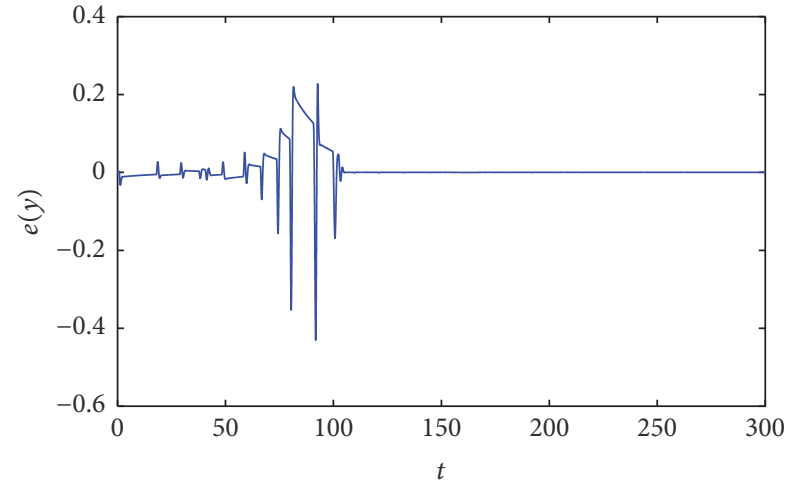

(b)

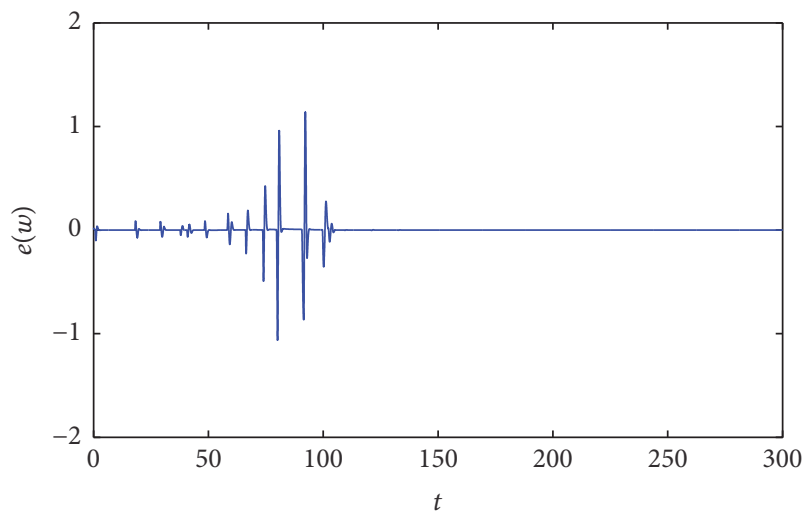

(d)

FIGURE 11: Graphs of time variation of the synchronization errors $e_{x}(t), e y(t), e_{z}(t)$, and $e_{w}(t)$.

$\alpha_{0}=0.05053, \alpha_{1}=0.15, \varepsilon=10, d=3.57, e=7.4$, and $b=0.69$ such that the chaotic state in Figure 3 is maintained and use the following initial conditions for the drive-response system: $\left(x_{1}, y_{1}, z_{1}, w_{1}\right)=(0.0201,0.001,0.002,0.011),\left(x_{2}\right.$, $\left.y_{2}, z_{2}, w_{2}\right)=(0.031,0.0051,0.012,0.001)$.
The graphs of Figure 11 illustrate the time evolutions of the synchronization errors. According to these graphs, one observes that the synchronization is reached, as the errors amplitudes are decreasing with time towards a small limit close to zero. Since Figure 11 presents the performance of the 


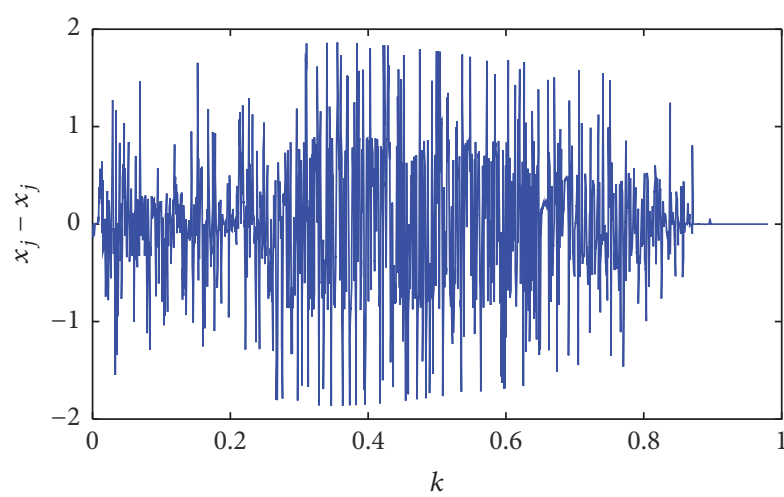

(a)

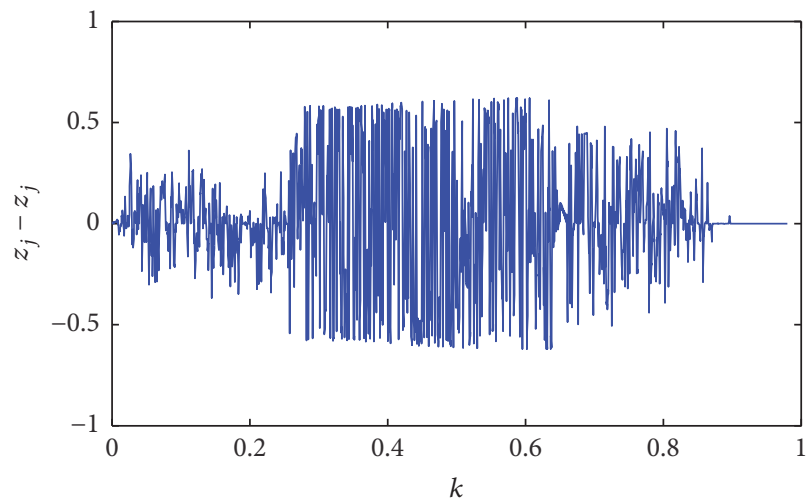

(c)

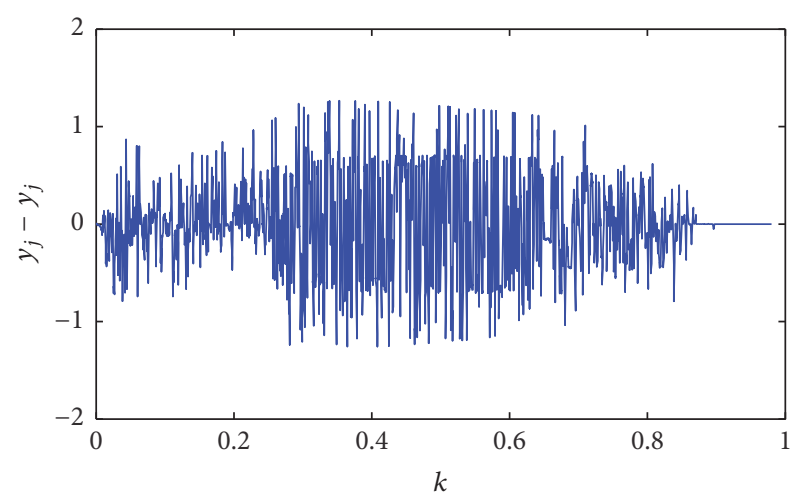

(b)

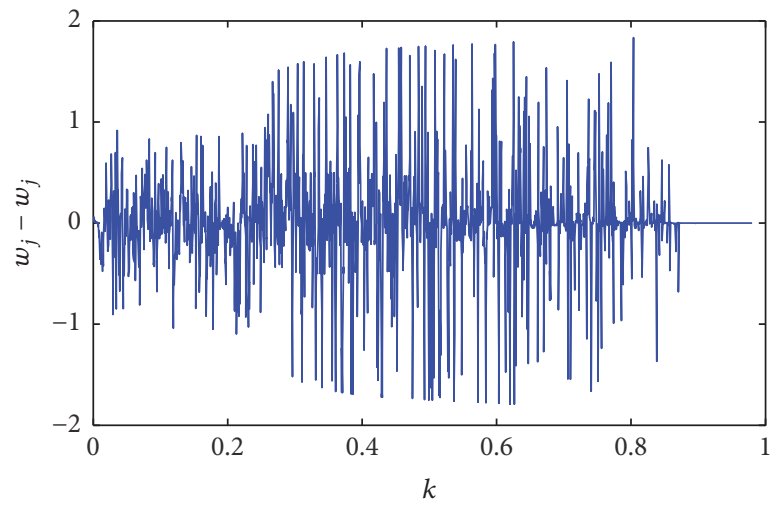

(d)

FIGURE 12: Bifurcation diagram for the in-phase synchronization, respectively, $x_{j}-x_{3-j}, y_{j}-y_{3-j}, z_{j}-z_{3-j}$, and $w_{j}-w_{3-j}$.

controller, the error dynamics are found to converge to the zero solution as $t \rightarrow \infty$, implying that the synchronization between systems (15) and (16) has been achieved.

Figure 12 presents the bifurcation diagrams, the synchronization boundaries of the system according to the coupling parameter $k$.

5.2. Experimental Study. The complex behavior of the system (Figure 1) has been experimentally investigated. Various bifurcation and the synchronization state are examined, when the distance between the magnetic core coils is monitored, while keeping all values of electronic components constant. It is important to mention that the analog voltages obtained from our circuit are directly the voltages $V_{C i}$ or $V_{C 2 j}$ of (1).

Figure 13 shows the photograph of the experimental Colpitts oscillators. This circuit is carried out using bipolar junction transistors (2N3904) and we set the following values: $V_{0}=10 \mathrm{~V}, R_{0}=35 \Omega, L_{j}=1.2 \mathrm{mH}, C_{1 j}=C_{2 j}=470 \mathrm{nF}$, $C_{3 j}=47 \mathrm{nF}, R_{3}=510 \Omega R_{b}=1 \Omega$, and $R_{1}=R_{2}=2 \mathrm{k} \Omega$.

Some examples are reported in Figure 14, in particular the synchronization between the oscillators obtained for $k \geq 0.9$.

\section{Conclusion}

The aim of this paper was to introduce a new method which is a simple, cheap, and easy technique for the study of

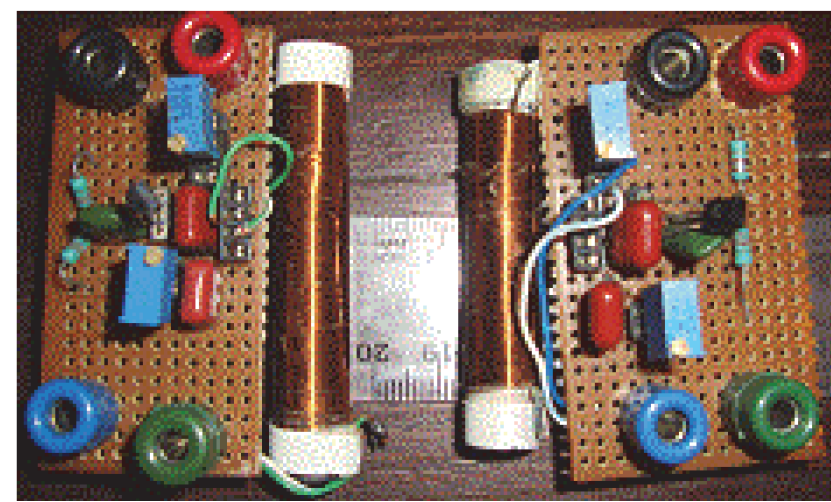

FIGURE 13: Experimental circuit.

dynamic and synchronization of Colpitts coupled oscillator. This method has gone through experiment and has been numerically characterized. The influence of the magnetic field via a magnetic coupling between the two oscillators has been presented. The stability of the fixed points has been investigated and the analytical formulas describing the stability of the system have been established. The bifurcation analysis of the phenomena leading to the creation of multistability of the system has been studied. Numerical simulations and electronic circuit implementation of the system show 
(1)
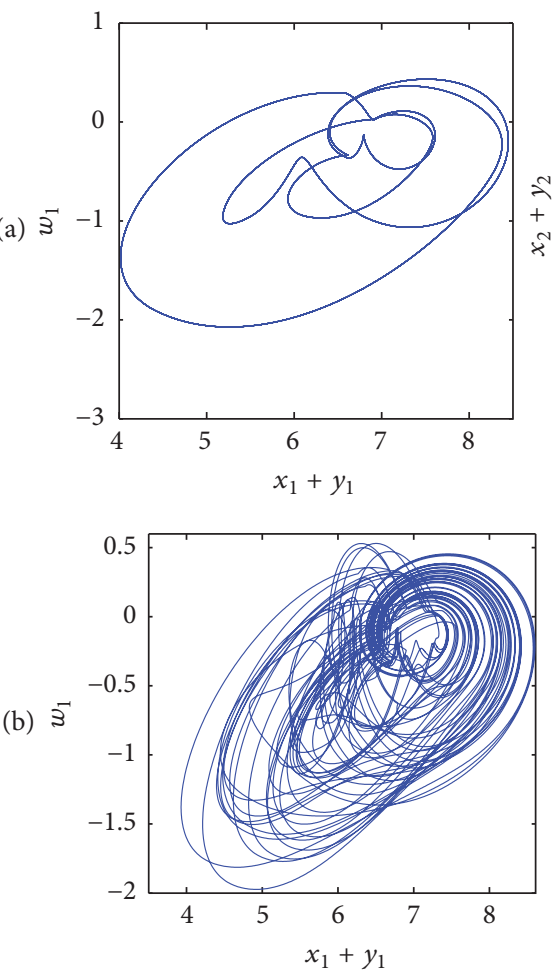

(c) ริ

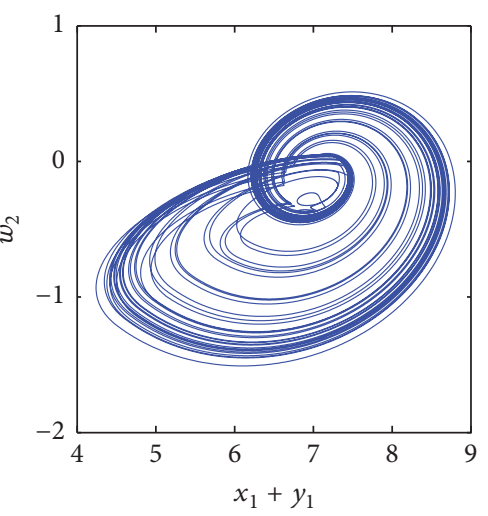

(2)
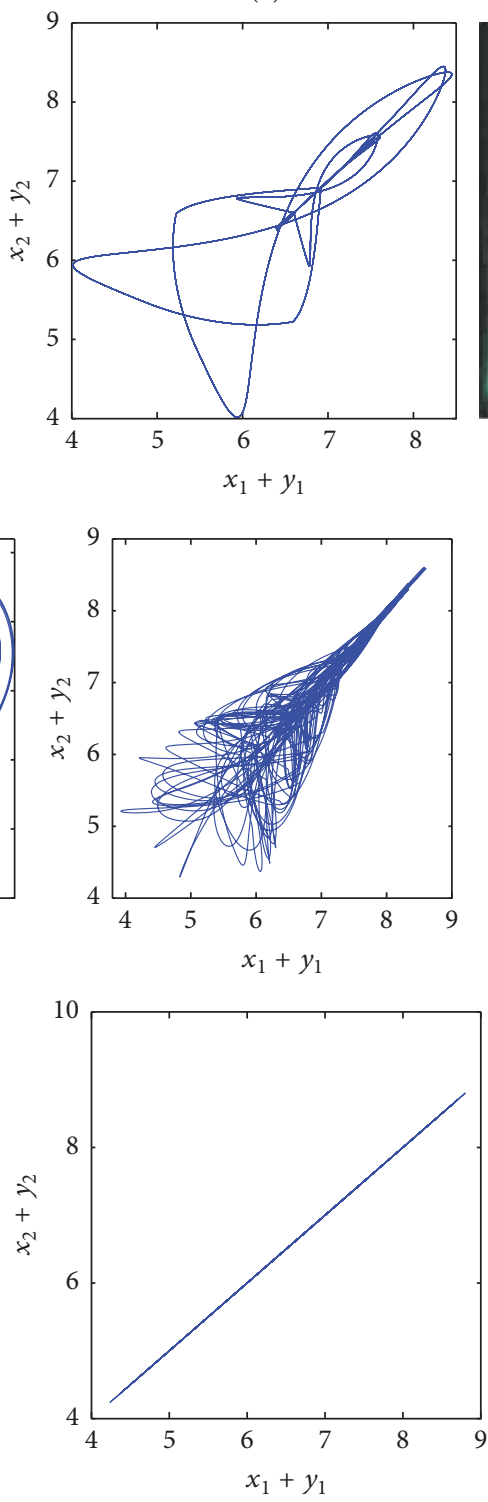

(3)
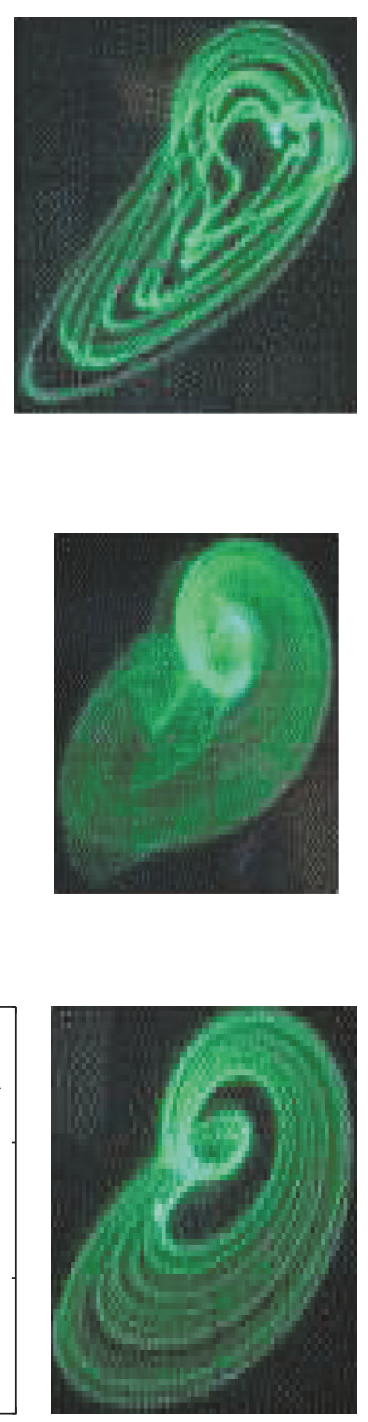

$(4)$
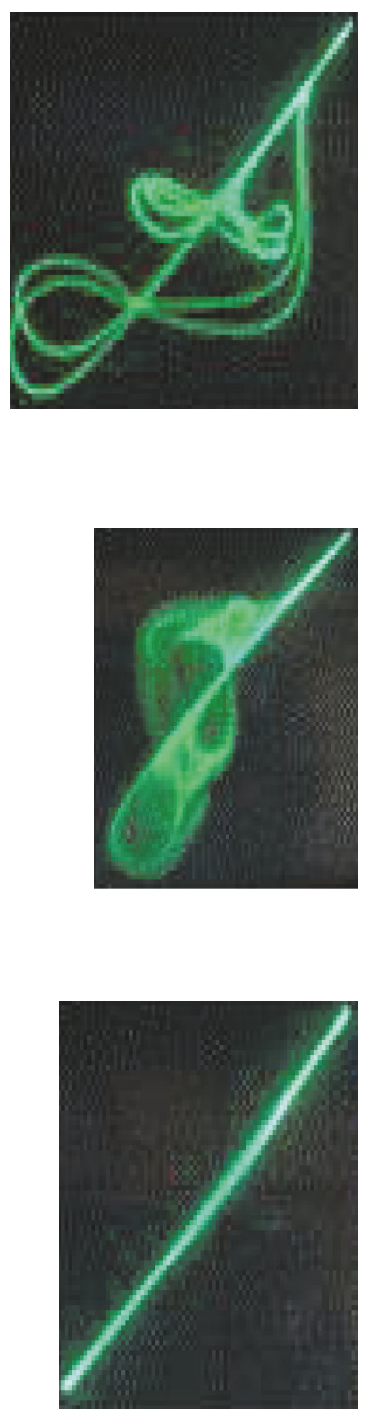

FIGURE 14: In-phase synchronization of the magnetically coupled chaotic circuits (a) $k=0.67$; (b) $k=0.8$; and (c) $k=0.9$. (1) $x_{1}+y_{1}$ versus $w_{1}$; (2) $x_{1}+y_{1}$ versus $x_{2}+y_{2}$; (3) $V_{C 11}$ versus $V_{L 1}$; (4) $V_{C 11}$ versus $V_{C 12}$.

that the complex dynamics (transient chaos and transient hyperchaos) of the circuit are heavily dependent on the initial state of this system and on the magnetic coupling factor $k$. We have shown that the magnetic coupling may be used to generate the hyperchaotic behavior. This can also drive two identical chaotic oscillators in a synchronized state as well as achieving the global stabilization of the system to its regular dynamics [15]. The dynamics of the coupled system have been investigated and Lyapunov stability theory has been applied to prove that, under some conditions, the drive-response system can achieve practical synchronization. The electronic circuit implementation has been realized to demonstrate the effectiveness of the controller. In future works, the following aspect will be considered: (i) the case of two nonidentical Colpitts oscillators and (ii) the collective dynamics of several magnetically coupled Colpitts systems.

\section{Conflicts of Interest}

The authors declare that there are no conflicts of interest regarding the publication of this paper.

\section{References}

[1] E.-W. Bai and K. E. Lonngren, "Synchronization of two Lorenz systems using active control," Chaos, Solitons and Fractals, vol. 8, no. 1, pp. 51-58, 1997.

[2] H. B. Fotsin and P. Woafo, "Adaptive synchronization of a modified and uncertain chaotic van der Pol-Duffing oscillator based on parameter identification," Chaos, Solitons and Fractals, vol. 24, no. 5, pp. 1363-1371, 2005.

[3] U. E. Vincent, R. K. Odunaike, J. A. Laoye, and A. A. Gbindinninuola, "Adaptive backstepping control and synchronization of a modified and chaotic Van der Pol-Duffing oscillator," Journal 
of Control Theory and Applications, vol. 9, no. 2, pp. 273-277, 2011.

[4] C. Li, X. Liao, and K.-W. Wong, "Lag synchronization of hyperchaos with application to secure communications," Chaos, Solitons \& Fractals, vol. 23, no. 1, pp. 183-193, 2005.

[5] M. P. Kennedy, "Chaos in the colpitts oscillator," IEEE Transactions on Circuits and Systems I: Fundamental Theory and Applications, vol. 41, no. 11, pp. 771-774, 1994.

[6] G. M. Maggio, O. De Feo, and M. P. Kennedy, "Nonlinear analysis of the Colpitts oscillator and applications to design," IEEE Transactions on Circuits and Systems I: Fundamental Theory and Applications, vol. 46, no. 9, pp. 1118-1130, 1999.

[7] J. Kengne, J. C. Chedjou, G. Kenne, and K. Kyamakya, "On the analysis of semiconductor diode-based chaotic," Nonlinear Dynamics, vol. 1071, pp. 1301-1309, 2014.

[8] O. E. Rössler, "An equation for hyperchaos," Physics Letters A, vol. 71, no. 2-3, pp. 155-157, 1979.

[9] T. Matsumoto, L. O. Chua, and K. Kobayashi, "Hyper chaos: laboratory experiment and numerical confirmation," IEEE Transactions on Circuits and Systems, vol. 33, no. 11, pp. 11431147, 1986.

[10] H. Zhang, X.-K. Ma, Y. Yang, and C.-D. Xu, “Generalized synchronization of hyperchaos and chaos using active backstepping design," Chinese Physics, vol. 14, no. 1, pp. 86-94, 2005.

[11] A. Čenys, A. Tamaševičius, A. Baziliauskas, R. Krivickas, and E. Lindberg, "Hyperchaos in coupled Colpitts oscillators," Chaos, Solitons and Fractals, vol. 17, no. 2-3, pp. 349-353, 2003.

[12] M. D. Shrimali, A. Prasad, R. Ramaswamy, and U. Feudel, “The nature of attractor basins in multistable systems," International Journal of Bifurcation and Chaos in Applied Sciences and Engineering, vol. 18, no. 6, pp. 1675-1688, 2008.

[13] S. Kim, S. H. Park, and C. S. Ryu, "Multistability in coupled oscillator systems with time delay," Physical Review Letters, vol. 79, pp. 1616-1619, 1997.

[14] U. Feudel, “Complex dynamics in multistable systems," International Journal of Bifurcation and Chaos in Applied Sciences and Engineering, vol. 18, no. 6, pp. 1607-1626, 2008.

[15] L. K. Kana, A. Fomethe, H. B. Fotsin, and P. H. Louodop, "A magnetic coupling based strategy for synchronization of a system consisting of chaotic modified van der pol dung oscillators," Journal of Nonlinear Dynamics, 2014.

[16] G. P. King and S. T. Gaito, "Bistable chaos. I. Unfolding the cusp," Physical Review A, vol. 46, no. 6, pp. 3092-3099, 1992.

[17] P. Ruiz, J. M. Gutiérrez, and J. Güémez, "Experimental mastering of nonlinear dynamics in circuits by sporadic pulses," Chaos, Solitons and Fractals, vol. 36, no. 3, pp. 635-645, 2008.

[18] A. Wolf, J. B. Swift, H. L. Swinney, and J. A. Vastano, "Determining Lyapunov exponents from a time series," Physica D: Nonlinear Phenomena, vol. 16, no. 3, pp. 285-317, 1985. 


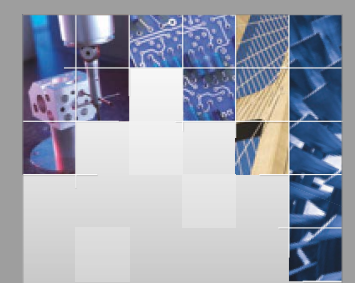

\section{Enfincering}
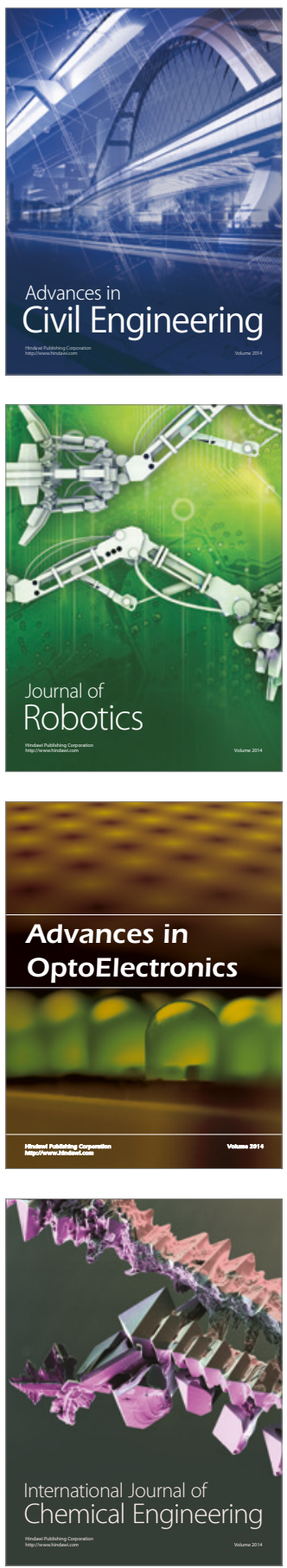

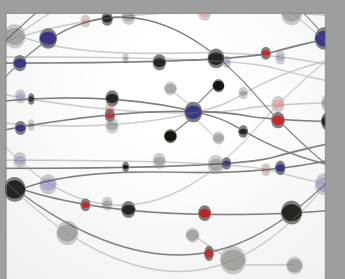

The Scientific World Journal

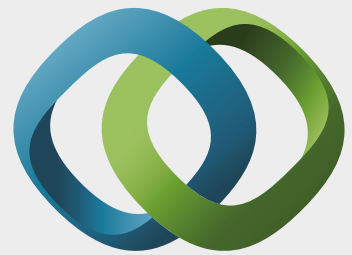

\section{Hindawi}

Submit your manuscripts at

https://www.hindawi.com
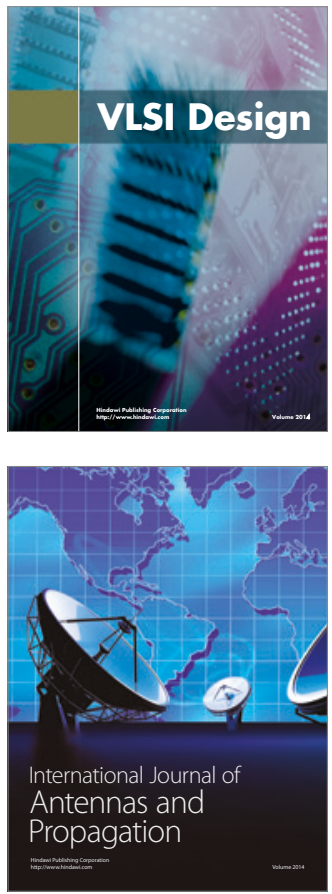

\section{Rotating}

Machinery
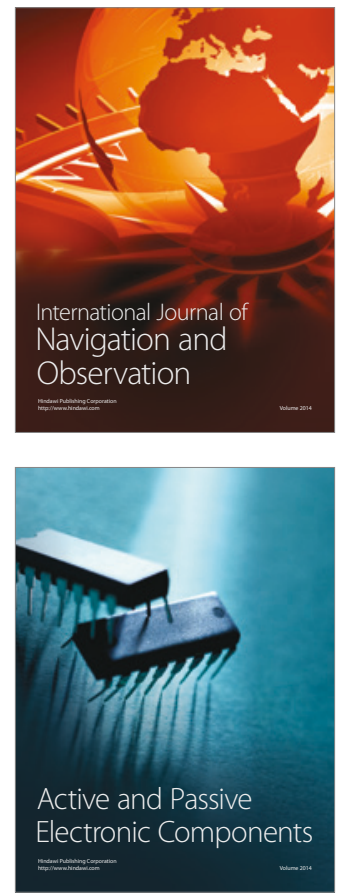
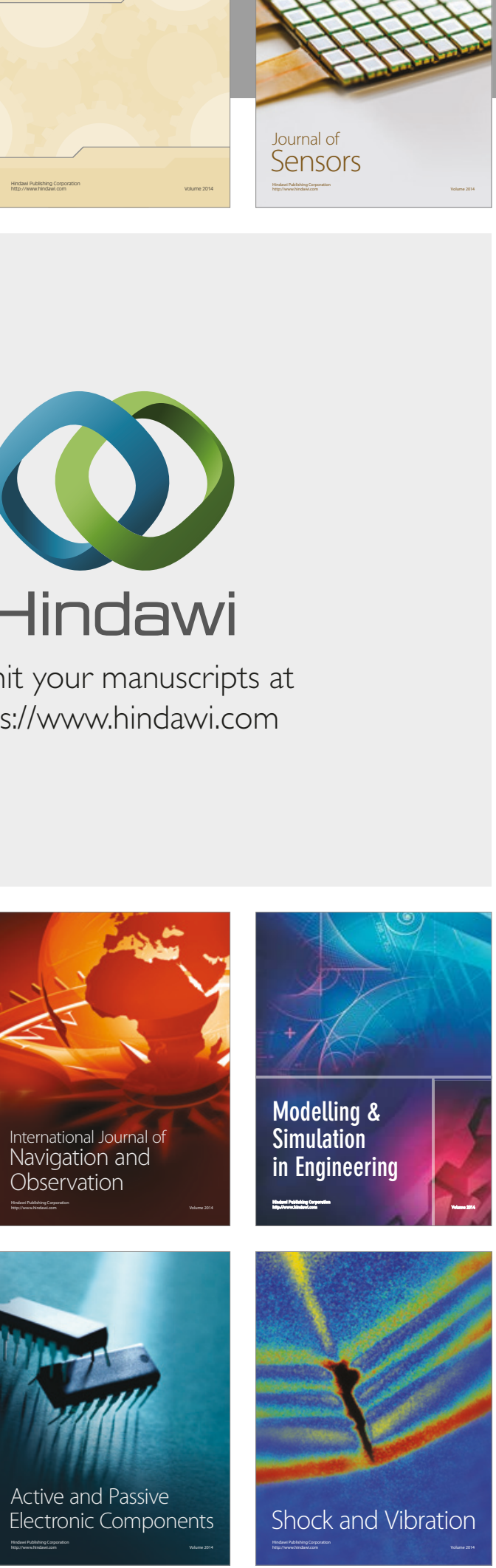
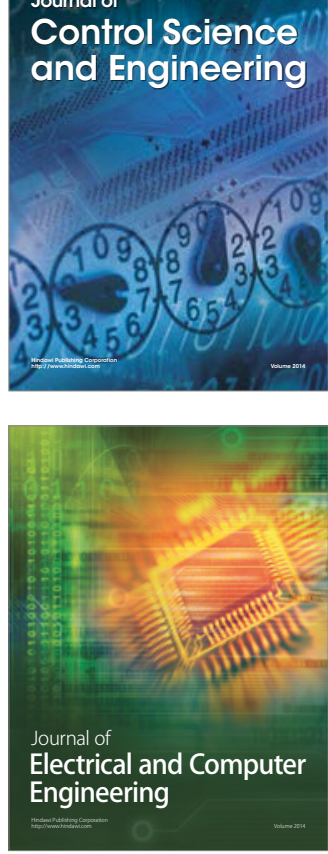

Distributed

Journal of

Control Science

and Engineering
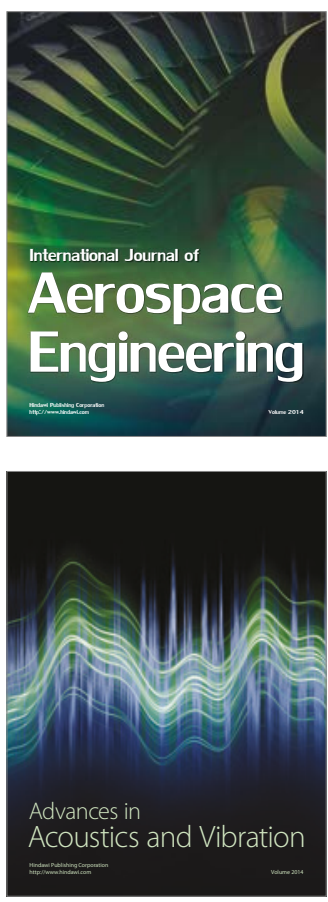

Sensor Networks 